\title{
Vaccine-preventable Diseases
}

\author{
1'Jaideep Malhotra, ${ }^{2}$ Ruchika Garg, ${ }^{3}$ Prashant Prakash, ${ }^{4}$ Manish Bansal
}

How to cite this article: Malhotra J, Garg R, Prakash P, Bansal M. Vaccine-preventable Diseases. J South Asian Feder Obst Gynae 2016;8(2):85-89.

\section{Source of support: Nil}

Conflict of interest: None

Date of received: 10 Feb 2016

Date of acceptance: 30 Mar 2016

Date of publication: April 2016

\section{INTRODUCTION}

Vaccine-preventable diseases are those diseases for which there is a shot that helps the immune system prepare for an infection. A person develops immunity after he or she has received a vaccine and responded to it. When a vaccinated person is exposed to a virus (for example, hepatitis B) or bacteria (for example, diphtheria), his or her body is able to destroy the virus or bacteria and prevent the disease. No vaccine is perfect, and some people who receive a vaccine can still get the disease. This is why it is important for everyone to get the vaccine. This gives the community what experts call "herd" immunity, which means that, basically, there are very few people who could serve as a reservoir for the disease. Herd immunity prevents severe outbreaks of a disease (Table 1).

\section{What is the Vaccination Schedule for Adolescents? (Table 2)}

\section{What is the Vaccination Schedule for Adults? (Tables 3 and 4)}

\section{Vaccination in Pregnancy}

The concern surrounding the dangers of vaccines during pregnancy are mostly theoretical. The concern is related to the risk of transfer of the disease to the fetus. There is no

\footnotetext{
${ }^{1}$ Consultant, ${ }^{2}$ Assistant Professor, ${ }^{3}$ Associate Professor ${ }^{4}$ Professor

${ }^{1}$ Rainbow IVF, Agra, Uttar Pradesh, India

2Department of Obstetrics and Gynecology, SN Medical College Agra, Uttar Pradesh, India

${ }^{3,4}$ Department of Medicine, SN Medical College, Agra, Uttar Pradesh, India

Corresponding Author: Ruchika Garg, Assistant Professor Department of Obstetrics and Gynecology, SN Medical College Agra, Uttar Pradesh, India, e-mail: ruchikagargagra@gmail.com
}

evidence of any risk associated with the use of inactivated (killed) vaccines in pregnant women. The biggest concern is related to the use of live vaccines. While the risk is extremely low, the concern is that the live virus will be transferred from the mother to the fetus. There must be a discussion between the physician and the mother whenever a live vaccine is considered during pregnancy. The CDC has issued a guide to help expectant mothers

Table 1: Vaccine-preventable diseases

\begin{tabular}{ll}
\hline Disease & Vaccine \\
\hline Diphtheria & Tetanus, diphtheria, and pertussis (Tdap) \\
Hepatitis A & Hepatitis A \\
Hepatitis B & Hepatitis B \\
Human papilloma & HPV (3 doses) \\
virus (HPV) & \\
Influenza & Annual influenza vaccine \\
Measles & Measles, mumps, and rubella (MMR) \\
Meningococcal & Meningococcal (two vaccines covering \\
& separate serogroups) \\
Mumps & MMR \\
Pertussis & Tdap \\
Pneumococcal & Pneumococcal (multiple vaccines covering \\
disease & different serogroups) \\
Polio & Inactivated polio vaccine (IPV) \\
Rubella & MMR \\
Tetanus & Tdap \\
Varicella & Varicella \\
\hline
\end{tabular}

Table 2: Recommended vaccination schedule for adolescents (not including catch-up vaccinations)

\begin{tabular}{ll}
\hline Vaccine & $\begin{array}{l}\text { Recommended age } \\
\text { for vaccination }\end{array}$ \\
\hline $\begin{array}{l}\text { Tetanus, diphtheria, } \\
\text { pertussis (Tdap) } \\
\text { Human papillomavirus } \\
\text { (HPV) (three doses) }\end{array}$ & $11-12$ years \\
$\begin{array}{l}\text { Meningococcal conjugate } \\
\text { vaccine (MCV4) }\end{array}$ & $11-12$ years (1st dose) \\
$\begin{array}{l}\text { Influenza (flu) } \\
\text { Pneumococcal }\end{array}$ & $\begin{array}{l}13-18 \text { years (2nd dose) } \\
\text { yearly }\end{array}$ \\
& $\begin{array}{l}\text { Recommended for some children } \\
\text { with certain medical conditions } \\
\text { (check with the child's physician) }\end{array}$ \\
& $\begin{array}{l}\text { Recommended if the child is } \\
\text { catching up on missed vaccines }\end{array}$ \\
Hepatitis A & \\
Hepatitis B & \\
Inactivated polio vaccine & \\
(IPV) & \\
Measles, mumps, rubella & \\
(MMR) & \\
Varicella &
\end{tabular}


Table 3: Recommended adult immunization schedule, by vaccine and age group

\begin{tabular}{|c|c|c|c|c|}
\hline Vaccine/age group & $27-49$ years & 50-59 years & $60-64$ years & $\geq 65$ years \\
\hline Tetanus, diphtheria, pertussis (Tdap) & \multicolumn{3}{|c|}{$\begin{array}{l}\text { Substitute one-time dose with tetanus and diphtheria } \\
(T d) \text {, then booster with } T d \text { every } 10 \text { years }\end{array}$} & $\mathrm{Td}$ booster every 10 years \\
\hline Human papilloma vaccine & 3 doses & & & \\
\hline Varicella & \multicolumn{3}{|c|}{2 doses } & \\
\hline Zoster & & & & 1 dose \\
\hline Measles, mumps, rubella & \multicolumn{3}{|c|}{1 or 2 doses } & 1 dose \\
\hline Influenza & \multirow{2}{*}{\multicolumn{3}{|c|}{1 or 2 doses }} & se annually \\
\hline Pneumococcal (polysaccharide) & & & & 1 doses \\
\hline Hepatitis A & \multicolumn{4}{|c|}{2 doses } \\
\hline Hepatitis B & \multicolumn{4}{|c|}{3 doses } \\
\hline Meningococcal & \multicolumn{4}{|c|}{1 or more doses } \\
\hline
\end{tabular}

Table 4: Various medical disorders and vaccines

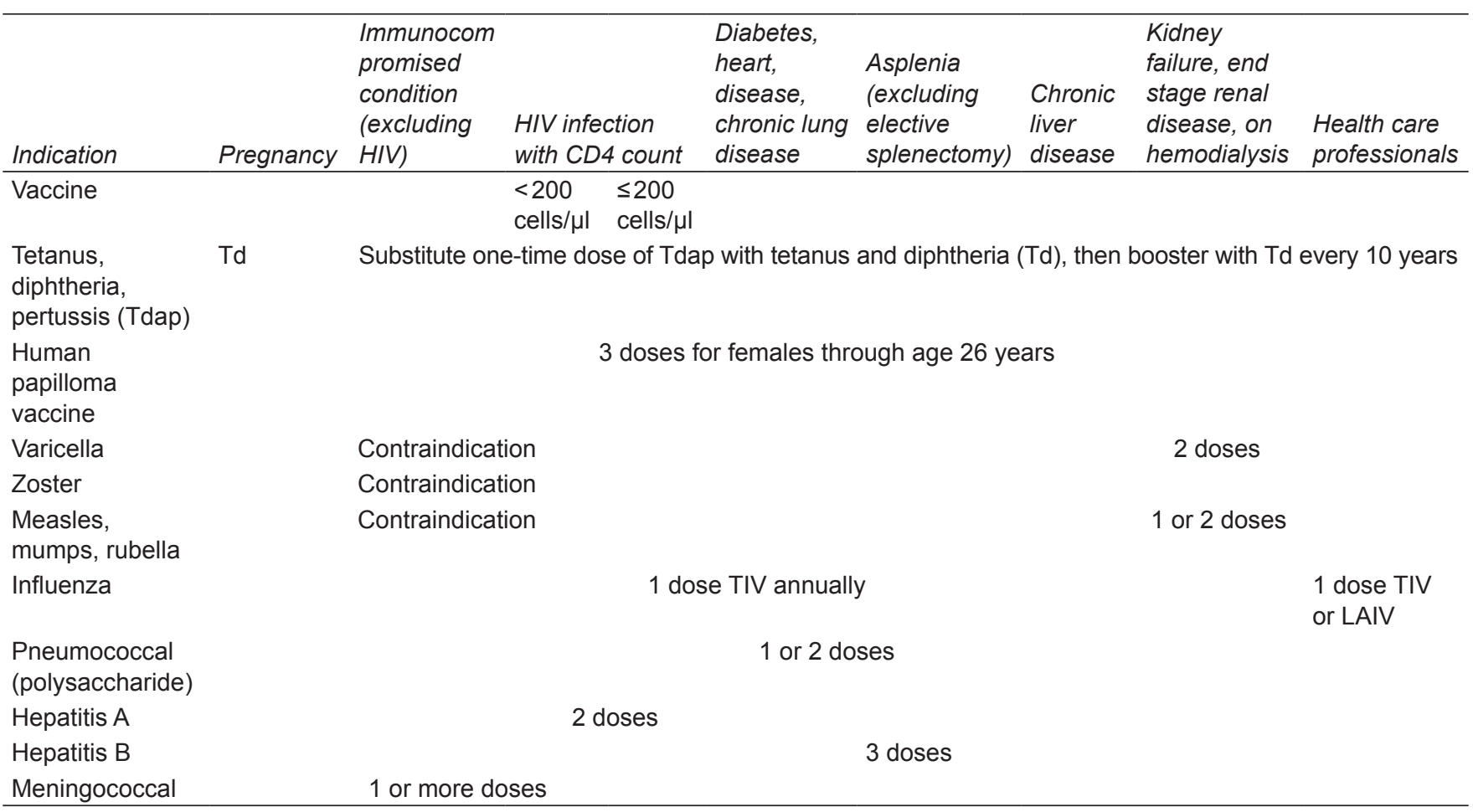

and physicians make good decisions related to the risk and benefits of using a vaccine during pregnancy. The tetanus-diphtheria and pertussis (Tdap) vaccine, hepatitis A and hepatitis B vaccine, meningococcal vaccine, and rabies vaccine are generally considered safe during pregnancy. It is currently recommended that pregnant women should not receive the following vaccines: HPV, live nasal influenza vaccine, MMR, varicella, and zoster.

\section{Do Health Care Workers need any Different Vaccines?}

It is very important that health care workers are upto-date on all of their required vaccines. Additionally, the $\mathrm{CDC}$ recommends that health care workers receive varicella vaccine unless there is proof of immunity, prior vaccination, or documented history of the disease. Also, health care workers with direct patient contact, who have not previously received a Tdap, are required to receive vaccine. Health care workers are required by most employers to have hepatitis B vaccination and annual influenza vaccinations.

\section{Tdap Vaccine and Who should receive It?}

Td and Tdap both contain vaccines against tetanus and diphtheria. Tetanus-diphtheria and pertussis also contains a vaccine against pertussis. Most adults are used to getting a tetanus shot when they get a cut. Up until 2005 , adolescents and adults were not recommended to receive the pertussis vaccine due to an unacceptable rate of negative reactions. In 2005, a new acellular pertussis vaccine became available for both children and adults. It is therefore recommended that all adolescents and adults 
Vaccine-preventable Diseases

receive at least one dose of Tdap when they are due for their next tetanus shot. Health care workers should get one Tdap vaccination as soon as possible but at least 2 years since the last tetanus shot (Td).

\section{What is the HPV Vaccine and Who should get It?}

The $\mathrm{CDC}$ recommends that all girls and boys receive the three-shot series beginning at 11 years of age. There was a change in recent years to reflect the fact that boys need vaccination in order to prevent spread to girls. Adult women who have not received the vaccine should do so up to 26 years of age. After 26 years of age, it is believed that most women would have been exposed to the virus and the vaccine would be of no use. In certain situations, such as abnormal Pap stain, known HPV infection, genital warts, and sexual assaults, HPV vaccine should be given. The vaccine induces high level of antibodies against HPV 16 and 18. The quadrivalent vaccine had an efficacy of less than $90 \%$ in preventing high-grade precancerous lesion of cervix. The duration of protection is not known. But it is usually more than 6 years. Booster doses are not advisable. Homosexual men are also recommended to take the vaccine.

\section{What is the Meningococcal Vaccine and Who should receive It?}

The disease is more common in adolescents and college students. Therefore, the meningococcal vaccine is recommended for all children between 11 and 12 years of age, with a booster dose at 16 years of age. If the child has not received the vaccine by 11 to 12 years of age, he or she should receive it up to age 18. College freshmen who have not received the vaccine should be vaccinated. The vaccines are also recommended for adults with special medical conditions.

\section{What is the MMR Vaccine and Who should receive It?}

The MMR vaccine contains vaccines against the disease measles, mumps, and rubella (German measles). These are all dangerous and potentially fatal diseases that have been successfully limited in the United States through aggressive vaccine programs. Adolescents and adults who have not received the MMR or the MMR plus varicella vaccine (MMRV) should receive two doses of the vaccine at least 1 month apart. People who do not have medical documentation of having had the disease or cannot prove previous vaccination should have titers (blood tests to check levels of immunity) drawn to make sure they are immune to these agents. If they do not have laboratory evidence of immunity, they should receive a two-dose series of vaccine.

\section{What is the Varicella Vaccine and Who should receive It?}

It is recommended that all adolescents and adults without any documented evidence of chicken pox or previous vaccination receive the two-dose series.

\section{What is the Pneumococcal Vaccine and Who should receive It?}

The vaccine is routinely given to children; however, it is given to only those adolescents and adults who are at a higher risk (patients with chronic diseases like heart disease and diabetes, immunocompromised individuals, smokers, and asthmatics). Elderly adults are one group that is considered at higher risk, and it is recommended that all adults receive one dose of the vaccine after 65 years of age. Two vaccines are available: 23 valent pneumococcal polysaccharide vaccine and 13 valent pneumococcal conjugate vaccine. Previously this vaccine was on controversy regarding its cost-effectiveness and efficacy. But at present, it is recommended that it be used in adults, especially with an underlying chronic disease, such as chronic obstructive pulmonary disease (COPD), asthma, renal disease, alcoholic, smoker, congestive heart failure, chronic liver disease, etc. A single dose of $0.5 \mathrm{~mL}$ administered by intramuscular (IM) or subcutaneous (SC) route in high-risk individuals can be repeated after 5 years.

\section{What is the Influenza Vaccine and Who should receive It?}

There are two types of influenza vaccines: A live-attenuated influenza vaccine (LAIV nasal spray) and an inactivated influenza vaccine (IIV). The IIV is administered as a single IM dose of $0.5 \mathrm{~mL}$. The LAIV is approved up to 50 years of age, while IIV is recommended to those above 50 years, to pregnant women, and to children between 6 months and 24 months. The current seasonal trivalent vaccine contains two influenza $A$ strains and one $B$ strain. The quadrivalent vaccine contains two influenza $\mathrm{A}$ and two B strains. The immunity from the vaccines is limited and vaccinations must be repeated yearly. In August 2008 , the advisory committee on immunization practices changed the recommendation on adolescents. It is now recommended that all people between 6 months and 18 years of age receive an annual vaccination. Although the CDC encourages that any adult who wants to decrease the chance of getting the flu get the influenza vaccine, certain high-risk groups are recommended to always get the annual flu vaccine. Adults over 50 years of age are considered to be at high risk and should receive the yearly influenza vaccination. Efficacy in adults less than 65 years is between 70 and $90 \%$, whereas in adults more than 65 years, it is less. 


\section{Can People with Egg Allergies still get the Influenza Vaccine?}

Most influenza vaccines are made using eggs. Therefore, people with severe egg allergy had previously been recommended not to receive the influenza vaccine. However, now there is a new vaccine, recombinant hemagglutinin influenza vaccine, which is not made using eggs. This vaccine is safe for patients with egg allergy.

\section{What is the Hepatitis A Vaccine and Who should receive It?}

Hepatitis A vaccine is routinely given to children; however, it is recommended only for certain highrisk adolescents and adults. Foreign travel is the most common reason for adults and adolescents to receive hepatitis A vaccination.

\section{What is the Hepatitis B Vaccine and Who should receive It?}

Hepatitis B vaccine is given routinely to children. Adolescents who did not receive their three-shot series as children should be given the vaccine. Adults are not routinely given the hepatitis $B$ vaccine unless they belong to certain high-risk groups. One high-risk group is health care workers. For immune competent adults, $20 \mu \mathrm{g}$ of recombinant vaccine is administered at 0,1 , and 6 months as an IM injection in the deltoid muscle. In chronic kidney disease (CKD) and immunocompromised persons, $40 \mu \mathrm{g}$ of recombinant vaccine is given at $0,1,2$, and 6 months. Routine post-vaccination screening is not advisable. But in high-risk individuals, after 1 to 2 months of last dose, protective concentration of anti-hepatitis B surface (HBs) should be measured. If concentration is less than $110 \mathrm{mlU} / \mathrm{mL}$, it is considered that immune response is inadequate. Nonresponders should be given a repeat full course of the vaccine, but after the initial response if the concentration declines then a booster dose is required.

\section{Zoster Vaccine}

In order to prevent the severe rash and the chronic pain, a vaccine (Zostavax) was developed and is recommended for all adults over 60 years of age who have no contraindication (pregnancy, severe immunocompromised state), including persons who report a previous episode of zoster, or who have chronic medical conditions (such as CKD, diabetes mellitus, COPD, etc.). The vaccine should be administered as a single dose of $0.65 \mathrm{~mL}$ via SC route in the deltoid region, which contains a minimum of 19,400 plaque-forming units of Oka/Merck strain of varicella Zoster virus.

\section{Yellow Fever Vaccine and Who should receive It?}

Travelers planning to visit endemic areas should receive the vaccine. It is a live-attenuated vaccine prepared from 17D YF virus. The vaccine is highly immunogenic and gives protection for more than 40 years. The dose is SC injection of $0.5 \mathrm{~mL}$ vaccine. Mild constitutional symptoms may appear, but fatal and serious adverse reactions (vaccine-associated neurotropic and viscerotropic) may happen, but are rare. The World Health Organization (WHO) warns against its use in pregnant females, immunocompromised hosts, post-thymectomy patients, people more than 60 years of age, and those who are allergic to eggs or chicken.

\section{Typhoid Vaccine}

Typhoid fever is an acute febrile illness caused by the bacterium Salmonella typhi. It is spread by contaminated food and water. Two types of vaccines - injectable Vi polysaccharide vaccine and live oral Ty21a vaccine are recommended for use. Live oral vaccine is orally administered. Four doses are given on alternate days. This series is repeated every 3 years. Oral vaccine should be taken with water. Foods should not be taken 1 hour after vaccination. Antibiotics should not be taken 3 days before and 7 days after vaccination. The vaccine elicits both serum and intestinal antibodies and cell-mediated immune response. The $\mathrm{Vi}$ vaccine is developed from $\mathrm{Vi}$ capsular polysaccharide of Ty2 S. typhi strain. The dose is $0.5 \mathrm{~mL} \mathrm{SC}$ or IM. Booster dose is recommended every 3 years. Protective effect ranges from 60 to $70 \%$. In 2013, Bharat serum launched a typhoid conjugate vaccine, Typhar-TCV ${ }^{\mathrm{TM}}$, which is a highly purified polysaccharide vaccine. It is the world's first vegetarian and nonbovine vaccine. Its long-term efficacy and safety have been proven.

Vaccines should be given to all adolescents. Routine adult vaccination is not recommended, but can be given to travelers, laboratory workers, household contact of S. typhi carriers, and whole community if there is an outbreak. Oral vaccine should not be used in pregnant women and HIV seropositive patients with CD4 count less than $200 / \mathrm{mm}^{3}$.

\section{Japanese Encephalitis Vaccine and Who should receive It?}

Japanese encephalitis (JE) is a mosquito-borne viral infection (flavivirus) that is a leading cause of encephalitis in Asia. This vaccine is primarily useful for children between 1 and 15 years. Recently, the government of India has made a strategy for its introduction in endemic areas and in states like West Bengal, Bihar, and Assam. Usually the vaccine is not recommended in adults except 
travelers to endemic areas and to laboratory workers. In children between 1 and 2 years it is given along with DPT booster doses. Primary hamster kidney (PHK) cell-cultured live-attenuated vaccine SA 14-14-2 is used in India in a dose of $0.5 \mathrm{~mL}$ given SC as a single dose. An indigenously produced JE vaccine was launched in
October 2013 This Vero cell-derived inactivated vaccine was developed jointly by the Indian Council of Medical Research (ICMR), the National Institute of Virology (NIV), and Bharat Biotech Ltd. Clinical trial showed efficacy of $96 \%$. The vaccine is applied as a $0.5 \mathrm{~mL}$ dose IM in a twodose schedule ( 0.28 days). 